\title{
Correlation Studies between Xanthophyll Yield and Other Parameters in Marigold
}

\author{
G. Swathi ${ }^{1 *}$ and B. Hemla Naik ${ }^{2}$ \\ ${ }^{1}$ Department of Floriculture and Landscape Architecture, College of Horticulture, \\ Mudigere, Chikmagalur District, Karnataka - 577 132, India \\ ${ }^{2}$ University of Agricultural and Horticultural Sciences, Shimoga, Karnataka-577 225, India \\ *Corresponding author:
}

\section{A B S T R A C T}

\begin{tabular}{|l|}
\hline Ke y w or d s \\
Marigold, VAM, \\
xanthophyll, \\
phosphorus, \\
Glomus fasciculatum, \\
G. mosseae, \\
G. intraradices. \\
\hline Article Info \\
\hline $\begin{array}{l}\text { Accepted: } \\
\text { 26 April } 2017 \\
\text { Available Online: } \\
\text { 10 May } 2017\end{array}$ \\
\hline
\end{tabular}

\section{Keywords}

Marigold, VAM xanthophyll, phosphorus,

G. mosseae,

intraradices.

\section{Introduction}

Marigold (Tagetes erecta L.) belongs to Asteraceae family and is a herbaceous plant with aromatic, pinnately divided leaves and is usually used as a bedding plant, cut flower, or as a coloring agent in poultry feed to obtain yellow egg yolks (Dole and Wilkins, 2005). $T$. erecta L. has smaller flowers and leaves than those of most other marigolds. The plants brighten up any sunny area in the landscape and attract attention. Moreover, marigold plants are considered a very valuable enter crop for controlling plant parasitic nematode as recorded by Basu and Roy (1975). The aerial parts of the plant contain high quality of essential oil that can be used for scenting soaps, perfumery, cosmetic, and pharmaceutical industries. Considering its importance as commercial flower crop, the study on effect of VAM fungi on the growth, yield and xanthophyll content of marigold, at different phosphorus levels was initiated.

\section{Materials and Methods}

The present investigation was conducted at experimental unit of Department of Floriculture and Landscape Architecture, 
College of Horticulture, Mudigere, Chikmagalur district, Karnataka during the period from October 2013 to February 2014 to know the correlation effect between xanthophyll yield and other parameters. A factorial experiment was laid out in Randomised Block Design.

There were 12 treatment combinations each three replications. In the present experiment VAM fungi (Glomus fasciculatum, $G$. mosseae, G. intraradices with an uninoculated control) and three levels of phosphorus $\left(60,90,120 \mathrm{~kg} \mathrm{ha}^{-1}\right)$ were tried in all possible combinations.
$\mathrm{M}_{1^{-}}$Glomus fasciculatum (Thaxter) Gerd. and Trappe.

$\mathrm{M}_{2^{-}}$Glomus mossea (Nicol. and Gerd.) Gerd. and Trappe.

$\mathrm{M}_{3^{-}}$Glomus intraradices Schenck and Smith.

$\mathrm{M}_{0}$ - Uninoculated control

Factor II $=$ Phosphorus levels 3

(225kg N $+60 \mathrm{~kg} \mathrm{~K} \mathrm{~K}_{2} \mathrm{O}$ as constant)

$\mathrm{P}_{1}-60 \mathrm{~kg} \mathrm{P}_{2} \mathrm{O}_{5} \mathrm{ha}^{-1}$

$\mathrm{P}_{2}-90 \mathrm{~kg} \mathrm{P}_{2} \mathrm{O}_{5}$ ha $^{-1}$

$\mathrm{P}_{3}-120 \mathrm{~kg} \mathrm{P}_{2} \mathrm{O}_{5} \mathrm{ha}^{-1}$

Factor $\mathbf{I}=$ Mycorrhizal species

\section{Treatment Combination}

\begin{tabular}{|l|l|l|}
\hline Treatment No. & Treatment & Combination \\
\hline $\mathrm{T}_{1}$ & $\mathrm{M}_{0} \mathrm{P}_{1}$ & Uninoculation $+60 \mathrm{~kg} \mathrm{P}_{2} \mathrm{O}_{5} \mathrm{ha}^{-1}$ \\
\hline $\mathrm{T}_{2}$ & $\mathrm{M}_{0} \mathrm{P}_{2}$ & Uninoculation $+90 \mathrm{~kg} \mathrm{P}_{2} \mathrm{O}_{5} \mathrm{ha}^{-1}$ \\
\hline $\mathrm{T}_{3}$ & $\mathrm{M}_{0} \mathrm{P}_{3}$ & Uninoculation $+120 \mathrm{~kg} \mathrm{P}_{2} \mathrm{O}_{5} \mathrm{ha}^{-1}$ \\
\hline $\mathrm{T}_{4}$ & $\mathrm{M}_{1} \mathrm{P}_{1}$ & G. fasciculatum $+60 \mathrm{~kg}_{2} \mathrm{O}_{5} \mathrm{ha}^{-1}$ \\
\hline $\mathrm{T}_{5}$ & $\mathrm{M}_{1} \mathrm{P}_{2}$ & G. fasciculatum $+90 \mathrm{~kg}_{2} \mathrm{O}_{5} \mathrm{ha}^{-1}$ \\
\hline $\mathrm{T}_{6}$ & $\mathrm{M}_{1} \mathrm{P}_{3}$ & G. fasciculatum $+120 \mathrm{~kg}_{2} \mathrm{O}_{5} \mathrm{ha}^{-1}$ \\
\hline $\mathrm{T}_{7}$ & $\mathrm{M}_{2} \mathrm{P}_{1}$ & G. mosseae $+60 \mathrm{~kg} \mathrm{P}_{2} \mathrm{O}_{5} \mathrm{ha}^{-1}$ \\
\hline $\mathrm{T}_{8}$ & $\mathrm{M}_{2} \mathrm{P}_{2}$ & G. mosseae $+90 \mathrm{~kg}_{2} \mathrm{O}_{5} \mathrm{ha}^{-1}$ \\
\hline $\mathrm{T}_{9}$ & $\mathrm{M}_{2} \mathrm{P}_{3}$ & G. mosseae $+120 \mathrm{~kg} \mathrm{P}_{2} \mathrm{O}_{5} \mathrm{ha}^{-1}$ \\
\hline $\mathrm{T}_{10}$ & $\mathrm{M}_{3} \mathrm{P}_{1}$ & G. intraradices $+60 \mathrm{~kg}_{2} \mathrm{O}_{5} \mathrm{ha}^{-1}$ \\
\hline $\mathrm{T}_{11}$ & $\mathrm{M}_{3} \mathrm{P}_{2}$ & G. intraradices $+90 \mathrm{~kg}_{2} \mathrm{O}_{5} \mathrm{ha}^{-1}$ \\
\hline $\mathrm{T}_{12}$ & $\mathrm{M}_{3} \mathrm{P}_{3}$ & G. intraradices $+120 \mathrm{~kg} \mathrm{P}_{2} \mathrm{O}_{5} \mathrm{ha}^{-1}$ \\
\hline
\end{tabular}

\section{Observations on morphological parameters}

\section{Plant height (cm)}

The plant height was measured from the ground level to the top of the plant.

\section{Number of braches per plant}

The number of primary as well as secondary branches was counted from individual plant and the average was worked out.

\section{Dry matter production (g/plant)}

Dry matter production was estimated at three different stages of the plant growth. Three plants were uprooted randomly from the net plot in each treatment. Then leaves, stem, and flowers were separated and oven dried at a temperature of $70{ }^{\circ} \mathrm{C}$, till it reached constant weight.

Dry matter accumulation in different parts of the plant at different stages were weighed and recorded in grams. The total dry matter 
production was calculated by adding dry matter accumulation in leaves, stem, flowers and roots of respective stages.

\section{Leaf area $(\mathrm{LA})\left(\mathrm{dm}^{2}\right)$}

The leaf area was estimated by disc method as suggested by Johnson (1967) at all the stages of growth. The leaf area was calculated by using the formula.

$\mathrm{LA}=\frac{\mathrm{Wa} \times \mathrm{A}}{\mathrm{Wd}}$

Where,

$\mathrm{LA}=$ Leaf area $\left(\mathrm{dm}^{2}\right)$

$\mathrm{Wa}=$ Weight of foliage (inclusive of 25 discs weight) (g)

$\mathrm{Wd}=$ Weight of 25 discs $(\mathrm{g})$

$\mathrm{A}=$ Area of $25 \mathrm{disc}\left(\mathrm{dm}^{2}\right)$

\section{Leaf area duration (days)}

Leaf area duration (LAD) is the integral of leaf area index (LAI) over the growth period (Watson, 1952). It was worked out for different stages of growth as per the formula suggested by Power et al., (1967).

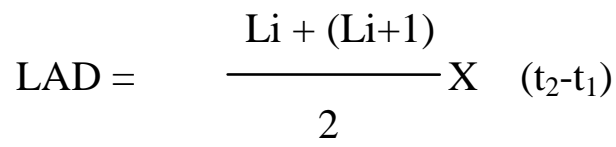

Where,

$\mathrm{LAD}=$ Leaf area duration (days)

$\mathrm{Li}=\mathrm{LAI}$ at an $\mathrm{i}^{\text {th }}$ Stage

$\mathrm{Li}+1=\mathrm{LAI}$ at $(\mathrm{I}+1)^{\mathrm{th}}$ stage

$\mathrm{T}_{2}-\mathrm{t}_{1}=$ Time interval between $\mathrm{i}^{\text {th }}$ and (I+1) stage in days.

\section{Crop growth rate (CGR) $\left(\mathrm{g} / \mathrm{m}^{2} / \mathrm{day}\right)$}

Crop growth rate is defined as the rate of dry matter production per unit ground per unit time (Watson, 1952) and was calculated by using the formula,

$$
\mathrm{CGR}=\frac{\mathrm{w}_{2}-\mathrm{w}_{1}}{\mathrm{t}_{2}-\mathrm{t}_{1}}
$$

Where,

CGR $=$ Crop growth rate $\left(\mathrm{g} / \mathrm{m}^{2} /\right.$ day $)$

$\mathrm{W}_{1}$ and $\mathrm{W}_{2}=$ Total dry matter production in grams $/ \mathrm{m}^{2}$ at time $\mathrm{t}_{1}$ and $\mathrm{t}_{2}$ respectively. $\mathrm{t}_{2}-\mathrm{t}_{1}=$ Time interval in days.

\section{Net assimilation rate (NAR)}

The NAR is the rate of dry weight increase per unit leaf area per unit time. It was calculated by following the formula given by Radford (1967) and expressed as g per unit leaf area per unit time

$$
\mathrm{NAR}=\frac{\left(\mathrm{W}_{2}-\mathrm{W}_{1}\right)}{\left(\mathrm{t}_{2}-\mathrm{t}_{1}\right)} \quad \mathrm{X} \frac{\left(\log _{\mathrm{e}} \mathrm{L}_{2}-\mathrm{Log}_{\mathrm{e}} \mathrm{L}_{1}\right)}{\left(\mathrm{L}_{2}-\mathrm{L}_{1}\right)}
$$

Where,

$\mathrm{L}_{1}$ and $\mathrm{W}_{1}=$ Leaf area $\left(\mathrm{cm}^{2}\right)$ and total dry weight of the plant $(\mathrm{g})$ respectively at time $t_{1}$ $\mathrm{L}_{2}$ and $\mathrm{W}_{2}=$ Leaf area $\left(\mathrm{cm}^{2}\right)$ and total dry weight of the plant $(\mathrm{g})$ respectively at time $t_{2}$.

\section{Observations on flowering and xanthophyll yield and its attributes}

\section{Flower size $(\mathbf{c m})$}

Ten fully opened flowers were selected randomly from the tagged plants and diameter was measured by using usual scale.

\section{Number of flowers per plant}

Number of flowers from each harvest was counted till the final harvest in the tagged plants and the average number of flowers per plant was worked out. 


\section{Flower yield per hectare (t/ ha)}

Flowers from plants other than tagged ones in net plot area were harvested separately and weighed treatment-wise. To this, flower weight of tagged plants was added to get net plot yield. Based on total net plot yield, yield per hectare was calculated.

\section{Petal meal yield per hectare (q)}

Petal meal yield per hectare was estimated based on the petal meal yield obtained per kilogram of fresh flower weight and it was multiplied by using the total flower yield per hectare and expressed as quintals per hectare.

\section{Xanthophyll estimation}

Xanthophyll was estimated by AOAC method (Lawrence, 1990).

\section{Nutrient analysis}

\section{Nitrogen estimation}

The estimation of nitrogen was done by Kjeldhal method as outlined by Jackson (1967).

\section{Total phosphorus (kg/ha)}

Digested plant sample with triacid mixture were used for estimation of phosphorus, and is expressed in kilogram per hectare.

It was estimated by Vanidomolybdate method as given by Jackson (1967) and the intensity of colour developed was read in spectrophotometer at $460 \mathrm{~nm}$.

\section{Uptake of phosphorous by plant}

Total phosphorous uptake was calculated for each treatment separately using the formula.
$\mathrm{P}$ uptake $=$

(kg/ ha)

Per cent of nutrient concentration X Biomass $(\mathrm{kg} / \mathrm{ha})$

100

And it was expressed in $\mathrm{kg}$ per hectare.

\section{Results and Discussion}

The correlation co-efficient (r) between xanthophyll yield per hectare and other parameters are presented in table 1 .

\section{Plant height}

Plant height was positively and significantly correlated with number of secondary branches $(\mathrm{r}=+0.990)$, total dry matter production $(\mathrm{r}=$ $+0.997)$, leaf area $(\mathrm{r}=+0.997)$, LAD $(\mathrm{r}=$ $+0.996)$, NAR $(\mathrm{r}=+0.996)$, CGR ( $\mathrm{r}=$ $+0.946)$, flower size $(\mathrm{r}=+0.851)$, number of flowers per plant $(\mathrm{r}=+0.950)$, flower yield per hectare $(\mathrm{r}=+0.916)$, petal meal yield per hectare $(\mathrm{r}=+0.945)$, N-uptake per hectare $(\mathrm{r}$ $=+0.979)$, P-uptake per hectare $(r=+0.982)$, xanthophyll content per $\mathrm{kg}$ of petal meal $(\mathrm{r}=$ $+0.948)$ and $x a n t h o p h y l l$ yield per hectare $(r=$ $+0.964)$.

\section{Number of secondary branches}

Number of secondary branches was positively and significantly correlated with plant height $(\mathrm{r}=+0.990)$, total dry matter production $(\mathrm{r}=$ $+0.998)$, leaf area $(\mathrm{r}=+0.998)$, LAD $(\mathrm{r}=$ $+0.998)$, NAR ( $\mathrm{r}=+0.999)$, CGR ( $\mathrm{r}=$ $+0.968)$, flower size $(\mathrm{r}=+0.902)$, number of flowers per plant $(\mathrm{r}=+0.944)$, flower yield per hectare $(\mathrm{r}=+0.919)$, petal meal yield per hectare $(\mathrm{r}=+0.935)$, N-uptake per hectare $(\mathrm{r}$ $=+0.980)$, P-uptake per hectare $(r=+0.984)$, xanthophyll content per $\mathrm{kg}$ of petal meal $(\mathrm{r}=$ $+0.953)$ and xanthophyll yield per hectare $(\mathrm{r}=$ +0.949 ). 
Table.1 Correlation studies between xanthophyll yield and other parameters

\begin{tabular}{|c|c|c|c|c|c|c|c|c|c|c|c|c|c|c|c|}
\hline Characters & 1 & 2 & 3 & 4 & 5 & 6 & 7 & 8 & 9 & 10 & 11 & 12 & 13 & 14 & 15 \\
\hline 1. Plant height & 1 & $0.990 * *$ & $0.997 * *$ & $0.997 * *$ & $0.996^{* *}$ & $0.996^{* *}$ & $0.946 * *$ & $0.851^{* *}$ & $0.950 * *$ & $0.916^{* *}$ & $0.945^{* *}$ & $0.979 * *$ & $0.982 * *$ & $0.948 * *$ & $0.964 * *$ \\
\hline $\begin{array}{l}\begin{array}{l}2 . \\
\text { Number of secondary } \\
\text { branches }\end{array} \\
\end{array}$ & & 1 & $0.998 * *$ & $0.998 * *$ & $0.998^{* *}$ & $0.999 * *$ & $0.968^{* *}$ & $0.902 * *$ & $0.944 * *$ & $0.919^{* *}$ & $0.935^{* *}$ & $0.980 * *$ & $0.984 * *$ & $0.953^{* *}$ & $0.949 * *$ \\
\hline $\begin{array}{l}\text { 3. Total dry matter } \\
\text { production }\end{array}$ & & & 1 & $1.000 * *$ & $1.000^{* * *}$ & $1.000 * *$ & $0.961 * *$ & $0.881 * *$ & $0.949 * *$ & $0.920^{* *}$ & $0.942 * *$ & $0.982 * *$ & $0.985^{* *}$ & $0.953 * *$ & $0.958 * *$ \\
\hline 4. Leaf area & & & & 1 & $1.000^{* * *}$ & $1.000^{* *}$ & $0.961 * *$ & $0.881 * *$ & $0.949 * *$ & $0.920^{* *}$ & $0.942^{* *}$ & $0.982 * *$ & $0.985^{* *}$ & $0.953 * *$ & $0.958^{* *}$ \\
\hline 5. LAD & & & & & 1 & $1.000^{* *}$ & $0.962 * *$ & $0.884 * *$ & $0.948^{* *}$ & $0.920^{* * *}$ & $0.941^{* *}$ & $0.982 * *$ & $0.985^{* *}$ & $0.953^{* *}$ & $0.958 * *$ \\
\hline 6. NAR & & & & & & 1 & $0.962 * *$ & $0.885^{* *}$ & $0.948^{* *}$ & $0.920^{* *}$ & $0.941^{* *}$ & $0.982 * *$ & $0.985^{* *}$ & $0.953 * *$ & $0.957 * *$ \\
\hline 7. CGR & & & & & & & 1 & $0.904 * *$ & $0.874 * *$ & $0.862^{* *}$ & $0.854^{* *}$ & $0.971 * *$ & $0.971 * *$ & $0.924 * *$ & $0.872 * *$ \\
\hline 8. Flower size & & & & & & & & 1 & $0.797 * *$ & $0.841^{* *}$ & $0.826^{* *}$ & $0.824 * *$ & $0.837^{* *}$ & $0.914 * *$ & $0.839 * *$ \\
\hline $\begin{array}{l}\text { 9. Number of flowers per } \\
\text { plant }\end{array}$ & & & & & & & & & 1 & $0.917^{* * *}$ & $0.936^{* *}$ & $0.921 * *$ & $0.937^{* *}$ & $0.925^{* *}$ & $0.954 * *$ \\
\hline 10. Flower yield per ha & & & & & & & & & & 1 & $0.949 * *$ & $0.874 * *$ & $0.876^{* *}$ & $0.934 * *$ & $0.952 * *$ \\
\hline $\begin{array}{l}\text { 11. Petal meal yield per } \\
\text { hectare }\end{array}$ & & & & & & & & & & & 1 & $0.893 * *$ & $0.900 * *$ & $0.919 * *$ & $0.982 * *$ \\
\hline 12. N-uptake per hectare & & & & & & & & & & & & 1 & $0.997 * *$ & $0.909 * *$ & $0.908^{* *}$ \\
\hline 13. P uptake per hectare & & & & & & & & & & & & & 1 & $0.923 * *$ & $0.919 * *$ \\
\hline $\begin{array}{l}\text { 14. Xanthophyll content per } \\
\mathrm{kg} \text { of petal meal }\end{array}$ & & & & & & & & & & & & & & 1 & $0.964 * *$ \\
\hline $\begin{array}{l}\text { 15. Xanthophyll yield per } \\
\text { hectare }\end{array}$ & & & & & & & & & & & & & & & 1 \\
\hline
\end{tabular}

**. Correlation is significant at the 0.01 level 


\section{Total dry matter production}

Total dry matter production was positively and significantly correlated with plant height $(r=+0.997)$, number of secondary branches ( $r$ $=+0.998)$, leaf area $(\mathrm{r}=+1.000)$, LAD $(\mathrm{r}=$ $+1.000)$, NAR $(\mathrm{r}=+1.000)$, CGR ( $\mathrm{r}=$ $+0.961)$, flower size $(\mathrm{r}=+0.881)$, number of flowers per plant $(\mathrm{r}=+0.949)$, flower yield per hectare $(\mathrm{r}=+0.920)$, petal meal yield per hectare $(\mathrm{r}=+0.942)$, N-uptake per hectare $(\mathrm{r}$ $=+0.982)$, P-uptake per hectare $(\mathrm{r}=+0.985)$, xanthophyll content per $\mathrm{kg}$ of petal meal $(\mathrm{r}=$ $+0.953)$ and xanthophyll yield per hectare $(\mathrm{r}=$ +0.958 ).

\section{Leaf area}

Leaf area was positively and significantly correlated with plant height $(\mathrm{r}=+0.997)$, number of secondary branches $(r=+0.998)$, total dry matter production $(\mathrm{r}=+1.000)$, LAD $(\mathrm{r}=+1.000)$, NAR $(\mathrm{r}=+1.000)$, CGR $(\mathrm{r}=$ $+0.961)$, flower size $(r=+0.881)$, number of flowers per plant $(\mathrm{r}=+0.949)$, flower yield per hectare $(\mathrm{r}=+0.920)$, petal meal yield per hectare $(\mathrm{r}=+0.942)$, N-uptake per hectare $(\mathrm{r}$ $=+0.982)$, P-uptake per hectare $(\mathrm{r}=+0.985)$, xanthophyll content per $\mathrm{kg}$ of petal meal $(\mathrm{r}=$ $+0.953)$ and xanthophyll yield per hectare $(\mathrm{r}=$ $+0.958)$.

\section{Leaf area duration (LAD)}

Leaf area duration was positively and significantly correlated with plant height $(\mathrm{r}=$ $+0.996)$, number of secondary branches $(r=$ $+0.998)$, total dry matter production ( $\mathrm{r}=$ $+1.000)$, leaf area $(\mathrm{r}=+1.000)$, NAR $(\mathrm{r}=$ $+1.000)$, CGR $(\mathrm{r}=+0.962)$, flower size $(\mathrm{r}=$ $+0.884)$, number of flowers per plant $(\mathrm{r}=$ $+0.948)$, flower yield per hectare $(\mathrm{r}=+0.920)$, petal meal yield per hectare $(\mathrm{r}=+0.941), \mathrm{N}$ uptake per hectare $(\mathrm{r}=+0.982)$, P-uptake per hectare $(\mathrm{r}=+0.985)$, xanthophyll content per $\mathrm{kg}$ of petal meal $(\mathrm{r}=+0.953)$ and xanthophyll yield per hectare $(r=+0.958)$.

\section{Net assimilation rate (NAR)}

Net assimilation rate was positively and significantly correlated with plant height $(\mathrm{r}=$ $+0.996)$, number of secondary branches $(r=$ $+0.999)$, total dry matter production $(\mathrm{r}=$ $+1.000)$, leaf area $(\mathrm{r}=+1.000)$, LAD $(\mathrm{r}=$ $+1.000)$, CGR $(\mathrm{r}=+0.962)$, flower size $(\mathrm{r}=$ $+0.885)$, number of flowers per plant $(\mathrm{r}=$ $+0.948)$, flower yield per hectare $(\mathrm{r}=+0.920)$, petal meal yield per hectare $(\mathrm{r}=+0.941), \mathrm{N}$ uptake per hectare $(\mathrm{r}=+0.982)$, P-uptake per hectare $(r=+0.985)$, xanthophyll content per $\mathrm{kg}$ of petal meal $(\mathrm{r}=+0.953)$ and $x a n t h o p h y l l$ yield per hectare $(\mathrm{r}=+0.957)$.

\section{Crop growth rate (CGR)}

Crop growth rate was positively and significantly correlated with plant height $(\mathrm{r}=$ $+0.946)$, number of secondary branches $(r=$ $+0.968)$, total dry matter production $(\mathrm{r}=$ $+0.961)$, leaf area $(\mathrm{r}=+0.961)$, LAD $(\mathrm{r}=$ $+0.962)$, NAR $(r=+0.962)$, flower size $(r=$ $+0.904)$, number of flowers per plant $(\mathrm{r}=$ $+0.874)$, flower yield per hectare $(\mathrm{r}=+0.862)$, petal meal yield per hectare $(\mathrm{r}=+0.854), \mathrm{N}-$ uptake per hectare $(\mathrm{r}=+0.971)$, P-uptake per hectare $(r=+0.971)$, xanthophyll content per $\mathrm{kg}$ of petal meal $(\mathrm{r}=+0.924)$ and $x a n t h o p h y l l$ yield per hectare $(\mathrm{r}=+0.872)$.

\section{Flower size}

Flower size was positively and significantly correlated with plant height $(\mathrm{r}=+0.851)$, number of secondary branches $(\mathrm{r}=+0.902)$, total dry matter production $(\mathrm{r}=+0.881)$, leaf area $(\mathrm{r}=+0.881)$, LAD $(\mathrm{r}=+0.884)$, NAR $(\mathrm{r}$ $=+0.885)$, CGR $(r=+0.904)$, number of flowers per plant $(\mathrm{r}=+0.797)$, flower yield per hectare $(\mathrm{r}=+0.841)$, petal meal yield per hectare $(\mathrm{r}=+0.826)$, N-uptake per hectare $(\mathrm{r}$ $=+0.824)$, P-uptake per hectare $(\mathrm{r}=+0.837)$, xanthophyll content per $\mathrm{kg}$ of petal meal $(\mathrm{r}=$ $+0.914)$ and xanthophyll yield per hectare $(r=$ +0.839 ). 


\section{Number of flowers per plant}

Number of flowers per plant was positively and significantly correlated with plant height $(r=+0.950)$, number of secondary branches ( $r$ $=+0.944)$, total dry matter production $(\mathrm{r}=$ $+0.949)$, leaf area $(\mathrm{r}=+0.949)$, LAD $(\mathrm{r}=$ $+0.948)$, NAR ( $\mathrm{r}=+0.948)$, CGR $(\mathrm{r}=$ $+0.874)$, flower size $(r=+0.797)$, flower yield per hectare $(\mathrm{r}=+0.917)$, petal meal yield per hectare $(\mathrm{r}=+0.936)$, $\mathrm{N}$-uptake per hectare $(\mathrm{r}$ $=+0.921)$, P-uptake per hectare $(\mathrm{r}=+0.937)$, xanthophyll content per $\mathrm{kg}$ of petal meal $(\mathrm{r}=$ $+0.925)$ and xanthophyll yield per hectare $(\mathrm{r}=$ $+0.954)$.

\section{Flower yield per hectare}

Flower yield per hectare was positively and significantly correlated with plant height $(\mathrm{r}=$ $+0.916)$, number of secondary branches ( $\mathrm{r}=$ $+0.919)$, total dry matter production $(\mathrm{r}=$ $+0.920)$, leaf area $(\mathrm{r}=+0.920)$, LAD $(\mathrm{r}=$ $+0.920)$, NAR $(\mathrm{r}=+0.920)$, CGR $(\mathrm{r}=$ $+0.862)$, flower size $(\mathrm{r}=+0.841)$, number of flowers per plant $(\mathrm{r}=+0.917)$, petal meal yield per hectare $(\mathrm{r}=+0.949)$, $\mathrm{N}$-uptake per hectare $(\mathrm{r}=+0.874)$, P-uptake per hectare $(\mathrm{r}=$ $+0.876)$, xanthophyll content per $\mathrm{kg}$ of petal meal $(r=+0.934)$ and xanthophyll yield per hectare $(\mathrm{r}=+0.952)$.

\section{Petal meal yield per hectare}

Petal meal yield per hectare was positively and significantly correlated with plant height $(\mathrm{r}=+0.945)$, number of secondary branches $(\mathrm{r}$ $=+0.935)$, total dry matter production $(\mathrm{r}=$ $+0.942)$, leaf area $(\mathrm{r}=+0.942)$, LAD $(\mathrm{r}=$ $+0.941)$, NAR $(\mathrm{r}=+0.941)$, CGR $(\mathrm{r}=$ $+0.854)$, flower size $(\mathrm{r}=+0.826)$, number of flowers per plant $(\mathrm{r}=+0.936)$, flower yield per hectare $(\mathrm{r}=+0.949)$, N-uptake per hectare $(\mathrm{r}=+0.893)$, P-uptake per hectare $(\mathrm{r}=$ +0.900 ), xanthophyll content per $\mathrm{kg}$ of petal meal $(\mathrm{r}=+0.919)$ and xanthophyll yield per hectare $(\mathrm{r}=+0.982)$.

\section{N-uptake per hectare}

N-uptake per hectare was positively and significantly correlated with plant height $(\mathrm{r}=$ $+0.979)$, number of secondary branches $(r=$ $+0.980)$, total dry matter production $(\mathrm{r}=$ $+0.982)$, leaf area $(\mathrm{r}=+0.982)$, LAD $(\mathrm{r}=$ $+0.982)$, NAR $(\mathrm{r}=+0.982)$, CGR $(\mathrm{r}=$ $+0.971)$, flower size $(\mathrm{r}=+0.824)$, number of flowers per plant $(\mathrm{r}=+0.921)$, flower yield per hectare $(\mathrm{r}=+0.874)$, petal meal yield per hectare $(\mathrm{r}=+0.893)$, Puptake per hectare $(\mathrm{r}=$ $+0.997)$, xanthophyll content per $\mathrm{kg}$ of petal meal $(\mathrm{r}=+0.909)$ and xanthophyll yield per hectare $(r=+0.908)$.

\section{P-uptake per hectare}

P-uptake per hectare was positively and significantly correlated with plant height $(\mathrm{r}=$ $+0.982)$, number of secondary branches $(\mathrm{r}=$ $+0.984)$, total dry matter production $(\mathrm{r}=$ $+0.985)$, leaf area $(\mathrm{r}=+0.985)$, LAD $(\mathrm{r}=$ $+0.985)$, NAR $(\mathrm{r}=+0.985)$, CGR $(\mathrm{r}=$ $+0.971)$, flower size $(\mathrm{r}=+0.837)$, number of flowers per plant $(\mathrm{r}=+0.937)$, flower yield per hectare $(\mathrm{r}=+0.876)$, petal meal yield per hectare $(r=+0.900)$, Nuptake per hectare $(r=$ $+0.997)$, xanthophyll content per $\mathrm{kg}$ of petal meal $(\mathrm{r}=+0.923)$ and $x a n t h o p h y l l$ yield per hectare $(\mathrm{r}=+0.919)$.

\section{Xanthophyll content per $\mathrm{kg}$ of petal meal}

Xanthophyll content per $\mathrm{kg}$ of petal meal was positively and significantly correlated with plant height $(\mathrm{r}=+0.948)$, number of secondary branches $(\mathrm{r}=+0.953)$, total dry matter production $(\mathrm{r}=+0.953)$, leaf area $(\mathrm{r}=$ $+0.953)$, LAD $(\mathrm{r}=+0.953)$, NAR $(\mathrm{r}=$ $+0.953)$, CGR $(r=+0.0 .924)$, flower size $(r=$ $+0.914)$, number of flowers per plant $(\mathrm{r}=$ $+0.925)$, flower yield per hectare $(\mathrm{r}=+0.934)$, petal meal yield per hectare $(\mathrm{r}=+0.919), \mathrm{N}$ uptake per hectare $(\mathrm{r}=+0.909)$, P-uptake per hectare $(\mathrm{r}=+0.923)$ and xanthophyll yield per hectare $(r=+0.964)$. 


\section{Xanthophyll yield per hectare}

Xanthophylls yield was positively and significantly correlated with plant height $(\mathrm{r}=$ $+0.964)$, number of secondary branches $(\mathrm{r}=$ $+0.949)$, total dry matter production $(\mathrm{r}=$ $+0.958)$, leaf area $(\mathrm{r}=+0.958)$, LAD $(\mathrm{r}=$ $+0.958)$, NAR $(\mathrm{r}=+0.957)$, CGR $(\mathrm{r}=$ $+0.872)$, flower size $(\mathrm{r}=+0.839)$, number of flowers per plant $(\mathrm{r}=+0.954)$, flower yield per hectare $(\mathrm{r}=+0.952)$, petal meal yield per hectare $(\mathrm{r}=+0.982)$, Nuptake per hectare $(\mathrm{r}=$ $+0.908)$, P-uptake per hectare $(\mathrm{r}=+0.919)$ and xanthophyll content per $\mathrm{kg}$ of petal meal $(r=+0.964)$.

The correlation studies among the different characters and with xanthophyll yield per hectare revealed that, xanthophyll yield was found positively and significantly associated with most of the vegetative, floral, yield and its attributing characters.

Among the parameters studied for correlation, P-uptake/ ha exhibited significantly positive correlation which clearly indicated that, phosphorus played an important role in maximization of vegetative characters which inturn increased floral characteristics and flower yield, ultimately the highest xanthophyll yield was obtained at the treatment inoculation with $G$. fasciculatum and $\mathrm{P}$ at $90 \mathrm{~kg} / \mathrm{ha}$.

In conclusion, xanthophyll yield was positively and significantly correlated with most of the vegetative, floral, yield and its attributing characters and the treatment inoculated with $G$. fasciculatum and $\mathrm{P}$ at 90 $\mathrm{kg} /$ ha recorded highest xanthophyll yield.

\section{References}

Basu, S. D. and Roy, S. K., 1975, Rotylenchulus sp. a new ecto parasitic nematode in ted soil. Two and Bud (22(1), (17) Em). In: Abst, C.F.H., Tocklia Experimental Station Horhat, Aaaaem, India, vol. 46. Breeding for Resistance to Fungal Pathogens. Canadian Journal of Botany 68, 10391044 (1976).

Dole, J. M. and Wilkins, H. F., 2005, Floriculture Principles and Species. Prentice-Hall Inc., USA, p. 1023.

JACKSON, M.L., 1967, Soil chemical analysis, Prentice Hall, India Private Limited, New Delhi. pp.183-192.

Johnson, R. E., 1967, Comparision of methods for estimating cotton leaf area. Agronomy Journal, 60: 1894-1895.

Lawrence, J.F., 1990, Determination of total xanthophyll and marigold oleoresin. Journal of Association of Official Analytical Chemists, 2: 970-975.

Power, J. F., Wills, W. O., Gunes, D. L. AND PEICHMAN, G. A., 1967, Effect of soil temperature, phosphorus and plant age on growth analysis of barley. Agronomy Journal, 59: 231-234.

Radford, J.G., 1967, Growth analysis formulae; their use and abuse. Crop Science, 7:171-175.

Watson, D. J., 1952, The Physiological basis of variation in yield. Advances in Agronomy, 4: 101-144

\section{How to cite this article:}

Swathi, G. and Hemla Naik, B. 2017. Correlation Studies between Xanthophyll Yield and Other Parameters in Marigold. Int.J.Curr.Microbiol.App.Sci. 6(5): 2846-2853. doi: https://doi.org/10.20546/ijcmas.2017.605.322 